\title{
Speed Breeding could Supercharge the Traditional Breeding to Hasten the Development of New Crop Varieties
}

\author{
Krishnananda P Ingle ${ }^{1 *}$, Priya Pardeshi ${ }^{2}$ and Gopal Narkhede ${ }^{3}$ \\ ${ }^{1}$ Biotechnology Centre, Department of Agricultural Botany, Dr. Panjabrao Deshmukh Krishi Vidyapeeth, India \\ ${ }^{2}$ Department of Agricultural Botany, Dr. Panjabrao Deshmukh Krishi Vidyapeeth, India \\ ${ }^{3}$ PhD Research scholar, International Crops Research Institute for Semi Arid Tropics, Pathancheru, Hyderabad, India \\ *Corresponding Author: Krishnananda P Ingle, PhD Research Scholar, Biotechnology Centre, Dr. Panjabrao Deshmukh Krishi \\ Vidyapeeth, Maharashtra (India).
}

Received: June 12, 2019; Published: June 25, 2019

DOI: 10.31080/ASAG.2019.03.0548

The traditional breeding takes a long period of 8-10 years of breeding cycle for the development of crop varieties. The environmental changes and over growing population raised a serious concern for global food security. The major constraint in the breeding of most of the plant species is the generation time. Scientists believe that technology must be needed to accelerate the generation time through significant reduction in the breeding cycles which could be possible through speed breeding which accelerated the generation advancement to develop more climate resistant strains with different genetic backgrounds and hasten the breeding cycles of the crops.

'Speed Breeding' involves the maximum exposure of LED lighting to plants' to enhance the plant growth and optimization of photosynthetic rate and thereby shortens the breeding cycles/ generation times of the crops. For example, in case of wheat, speed breeding allows six generations per year compared to traditional breeding platform. Speed breeding significantly shortened the generation time, plant anatomy; plant-pathogen interaction and flowering time can be studied using this technology [1].

Speed breeding superseded "shuttle breeding", which allowed the planting of only two generations annually. Recently, it was reported that for durum wheat, bread wheat and for chickpea; speed breeding greatly reduces the generation time and helps in achieving six generations per year, while for Canola four generations could be taken [2]. Speed breeding technology can be combined with other several new generation technologies such as CRISPR gene editing, genomic selection and marker-assisted breeding etc. to get the end result faster [3]. The integration of speed breeding with other modern crop breeding technologies will revolutionized the development of new crop varieties and having a great potential to accelerate the cereal research and cultivar improvement with desired traits within a short generation time.

\section{Bibliography}

1. https://www.sciencedaily.com/releases/2018/01/1801011 44758. htm

2. Watson A. et al. "Speed breeding is a powerful tool to accelerate crop research and breeding". Nature Plants 4 (2018): 2329.

3. https://www.jic.ac.uk/news-and-events/news/2018/01/ speed-breeding/

Volume 3 Issue 7 July 2019

(C) All rights are reserved by Krishnananda P Ingle., et al. 